\title{
Discrete unified gas kinetic scheme on unstructured meshes
}

\author{
Lianhua Zhu ${ }^{\mathrm{a}}$, Zhaoli Guo ${ }^{\mathrm{a}, *}, \mathrm{Kun} \mathrm{Xu}^{\mathrm{b}}$ \\ ${ }^{a}$ State Key Laboratory of Coal Combustion, Huazhong University of Science and \\ Technology, Wuhan, 430074, China \\ ${ }^{b}$ Department of Mathematics, Hong Kong University of Science and Technology, Clear \\ Water Bay, Hong Kong, China
}

\begin{abstract}
The recently proposed discrete unified gas kinetic scheme (DUGKS) is a finite volume method for multiscale flow computations with asymptotic preserving property. The solution of the Boltzmann model equation is directly used for the construction of numerical flux and makes the scheme applicable in all flow regimes. In previous applications of the DUGKS, structured meshes have been mostly employed, which may have difficulties for problems with complex geometries. In this paper we will extend the DUGKS to unstructured meshes, with the implementation of computational fluid dynamics techniques to the DUGKS. Several test cases, i.e., the cavity flow ranging from continuum to free molecular regimes, a multiscale flow problem between two connected cavities with large pressure and density variations, high speed flows past multiple cylinders in slip and transitional regimes, and an impulsive start problem are performed, and the results are compared with the well-defined Direct Simulation Monte Carlo (DSMC) or Navier-Stokes (NS) solutions in their applicable regimes. The numerical results demonstrate the effectiveness of the proposed DUGKS for the study of multiscale flow problems.
\end{abstract}

Keywords: Kinetic equation, Unstructured mesh, Shakhov model

*Corresponding author

Email addresses: 1hzhu@hust.edu.cn (Lianhua Zhu), zlguo@hust.edu.cn (Zhaoli Guo), makxu@ust.hk (Kun Xu)

Preprint submitted to Computers \& Fluids

January 4, 2016

(C) 2016. This manuscript version is made available under the Elsevier user license http://www.elsevier.com/open-access/userlicense/1.0/ 


\section{Introduction}

Gas flows can be classified into different flow regimes based on the Knudsen number $(\mathrm{Kn})$, which is defined as the ratio of the mean free path of the gas to the physical characteristic length. For flow with $\mathrm{Kn}>0.001$, non-equilibrium 5 effects become important and the classical Navier-Stokes-Fourier (NSF) equations fail to describe such a flow [1, while the Boltzmann equation can serve as a fundamental equation which is valid in the whole range of Knudsen numbers.

There are mainly two types of numerical approaches to solve the Boltzmann equation. The first one is the widely used direct simulation Monte Carlo (DSMC) method [1, which is the prevailing technique for simulating high-speed rarefied gas flows. However, the DSMC is a single scale method, where the particle transport and collision processes are decoupled. As a result, the cell size and time step are required to be smaller than the mean free path and the mean particle collision time [1]. For flows in near continuum or continuum regime, this requirement will lead to enormous computational costs. Another undesired feature of the DSMC is the statistical noise that must be reduced through intensive sampling and averaging, which is more serious for low speed and small temperature variation flows [1]. Great efforts have been devoted to reduce statistical noise of the DSMC method 2, 3. The second approach for solving the Boltzmann equation is to use deterministic numerical schemes, i.e., the Discrete Velocity Methods (DVM) 4, 5, 6. Most DVM schemes are single scale methods with decoupled particle transport and collision, which have the same constraints on the time step and cell size as the DSMC [7, 8, 9]. Recently, some asymptotic preserving (AP) schemes have been proposed to overcome these disadvantages (e.g., [10, 11, 12]). The AP schemes for Boltzmann equation are designed to reduce to the appropriate discretization schemes for hydrodynamic equations automatically as the Knudsen number goes to zero, without resolving the mean free path and particle collision time on the computational grids. The AP schemes also treats the collision term implicitly using efficient man- 
zero. Their stability is independent of the Knudsen number. These schemes are able to recover the Euler solutions in the continuum limit, but it is still unclear whether the Navier-Stokes solutions can be accurately obtained [13.

Recently, a unified gas kinetic scheme (UGKS) has been constructed for all 35 Knudsen number flows [14, 15, 16, 17]. The UGKS is an aggressive enxtension of the gas kinetic scheme (GKS) which is a flux solver for hydrodynamic equations and is mainly used to simulate continuum flows [18. In the UGKS, the particle transport and collision effects are coupled in UGKS when updating the discrete distribution function. Consequently, the restrictions on the cell size and time

40 step are avoided, and the UGKS solutions depend on the ratio of the local time step to the particle collision time [13].

An alternative unified kinetic method, i.e., the discrete unified gas kinetic scheme (DUGKS), has been proposed for multi-regime flow computations recently [19, 20]. The DUGKS shares the same modeling mechanism as the

${ }_{45}$ UGKS 19. The main difference between the UGKS and the DUGKS lies in the construction of numerical flux for the discrete distribution function at cell interface. In UGKS, the flux is obtained from the time-dependent distribution function at the cell interface, and this solution is based on the local analytical integral solution of the model kinetic equation. While in DUGKS, the flux is calculated from the distribution function at a half time step, and this solution is determined from a numerical characteristic solution of the model kinetic equation. The flux in DUGKS couples the effects of particle transport and collision, and the updating rule is much simpler than the UGKS.

The DUGKS shares some similarities with the well-known lattice Boltzmann method (LBM) which can be viewed as a special discrete velocity type method. Both of them are based on the relaxation-type collision models, and employ the implicit-to-explicit transformation. The idea behind the flux evaluation method in the DUGKS is conceptually very similar to the streaming step of the LBM, i.e., the particles arrived at the cell face (in the DUGKS) or lattice node (in ${ }_{60}$ the LBM) are assumed to have streamed from the upwind, with collision effect considered. However, there are considerable differences between the DUGKS 
and the LBM. The LBM is essentially an Navier-Stokes equation solver under the low Mach number condition, thus can only be used to simulating near incompressible continuum flows. The DUGKS employing the LBM type discrete velocity set (i.e., the DnQm lattices) can be viewed as a finite-volume based off-lattice Boltzmann method. Their relative performances for continuum flows have been compared in 21. It is also found that the DUGKS is not a straight forward translation of the finite-difference interpreted LBM [22].

In previous works [19, 20, the DUGKS has been applied to both low speed and high speed non-equilibrium flows using structured meshes. However, most non-equilibrium flow problems involve complicated geometries, such as those in the microelectromechanical systems (MEMS) industrial and aerospace engineering. The use of unstructured mesh is preferable. In this work we aim to extend the DUGKS to unstructured meshes and demonstrate its effectiveness for the multiscale non-equilibrium flows.

The rest of the paper is organized as following. In Sec. 2, the general procedure of the DUGKS on unstructured meshes is presented. In Sec. 3, several numerical examples, including the micro cavity flow, an expansion flow between two connected cavities, and the rarefied gas flow passing through a single and double circular cylinders, will be computed to demonstrate the capability of the current method in simulating flows in different regimes. An additional test case, the impulsive start plate problem is used to verify the uniform convergence rate of DUGKS. A brief summary is given in the last section.

\section{Discrete unified gas kinetic scheme}

\subsection{Shakhov model}

The DUGKS is based on the Boltzmann model equation. In this study, the collision operator is approximated by the Shakhov model [23] for monatomic gases. In $D$ dimensional space, the model equation is

$$
\frac{\partial f}{\partial t}+\boldsymbol{\xi} \cdot \nabla f=-\frac{1}{\tau}\left[f-f^{S}\right],
$$


where $f=f(\boldsymbol{\xi}, \boldsymbol{\eta}, \boldsymbol{x}, t)$ is the velocity distribution function of particles with velocity $\boldsymbol{\xi}=\left(\xi_{1}, \ldots, \xi_{D}\right)$ in $D$ dimensional velocity space at position $\boldsymbol{x}=$ $\left(x_{1}, \ldots, x_{D}\right)$ and time $t$. Here $\boldsymbol{\eta}=\left(\xi_{D+1}, \ldots, \xi_{3}\right)$ is a vector in a space with dimension $L=3-D$, which accounts for the degrees of freedom other than the D-dimensional translational ones. $f^{S}$ is the Shakhov equilibrium distribution function given by the Maxwellian distribution function $f^{e q}$ plus a heat flux correction term

$$
\left.f^{S}=f^{e q}\left[1+(1-\operatorname{Pr}) \frac{\boldsymbol{c} \cdot \boldsymbol{q}}{5 p R T}\left(\frac{c^{2}+\eta^{2}}{R T}-5\right)\right)\right]=f^{e q}+f_{P r},
$$

where Pr is the Prandtl number and $\boldsymbol{c}=\boldsymbol{\xi}-\boldsymbol{U}$ is the peculiar velocity around the averaged macroscopic fluid velocity $\boldsymbol{U} ; \boldsymbol{q}$ is the heat flux, $R$ is the specific gas constant, and $T$ is the temperature. The collision time $\tau$ in Eq. (1) is related to the dynamic viscosity $\mu$ and pressure $p$ by $\tau=\mu / p$. The dynamic viscosity $\mu$ depends on temperature as

$$
\mu=\mu_{\mathrm{ref}}\left(\frac{T}{T_{\mathrm{ref}}}\right)^{\omega},
$$

where $\mu_{\text {ref }}$ is the viscosity at the reference temperature $T_{\text {ref }}$, and the exponent $\omega$ is a constant depends on the inter-molecular interaction model. The viscosity $\mu_{\text {ref }}$ can be related to the reference mean free path $\lambda_{\text {ref }}$. By using the Knudsen $(\mathrm{Kn})$, Mach (Ma) and Reynolds (Re) numbers, the $\mu_{\text {ref }} \sim \lambda_{\text {ref }}$ relation leads to (Eq. 1.29 in 24]),

$$
\mathrm{Kn}=\sqrt{\frac{2 \gamma}{\pi}} \frac{(5-2 \omega)(7-2 \omega)}{15} \frac{\mathrm{Ma}}{\mathrm{Re}}
$$

where $\gamma$ is the heat capacity ratio. The Kn, Ma and Re numbers are define as

$$
\mathrm{Kn}=\frac{\lambda_{\text {ref }}}{L_{\text {ref }}}, \quad \mathrm{Ma}=\frac{U_{\text {ref }}}{\sqrt{\gamma R T_{\text {ref }}}}, \quad \mathrm{Re}=\frac{\rho_{\text {ref }} U_{\text {ref }} L_{\text {ref }}}{\mu_{\text {ref }}},
$$

where $L_{\text {ref }}, U_{\text {ref }}, \rho_{\text {ref }}$ are the referenced length, velocity and density, respectively.

The Maxwellian distribution function $f^{e q}$ is given by

$$
f^{e q}=\frac{\rho}{(2 \pi R T)^{3 / 2}} \exp \left(-\frac{c^{2}+\eta^{2}}{2 R T}\right)
$$


where $\rho$ is the gas density. The conservative flow variables $\boldsymbol{W} \equiv(\rho, \rho \boldsymbol{U}, \rho E)^{T}$ are calculated as moments of the distribution function,

$$
\boldsymbol{W}=\int \boldsymbol{\psi} f \mathrm{~d} \boldsymbol{\xi} \mathrm{d}
$$

here $\boldsymbol{\psi}=\left(1, \boldsymbol{\xi}, \frac{1}{2}\left(\xi^{2}+\eta^{2}\right)\right)^{T}$ and $\rho E=\frac{1}{2} \rho U^{2}+C_{\mathrm{V}} T=\frac{1}{2} \rho U^{2}+p /(\gamma-1)$, where $C_{\mathrm{V}}$ is the heat capacity. The heat flux $\boldsymbol{q}$ is defined by

$$
\boldsymbol{q}=\frac{1}{2} \int \boldsymbol{c}\left(c^{2}+\eta^{2}\right) f \mathrm{~d} \boldsymbol{\xi} \mathrm{\eta} .
$$

The parameter $\boldsymbol{\eta}$ can be viewed as internal degree of freedom, and the dependence of $f$ on $\boldsymbol{\eta}$ can be removed by using two reduced distribution functions [25]

$$
\begin{aligned}
g(\boldsymbol{x}, \boldsymbol{\xi}, t) & =\int f(\boldsymbol{\xi}, \boldsymbol{\eta}, \boldsymbol{x}, t) \mathrm{d} \boldsymbol{\eta}, \\
h(\boldsymbol{x}, \boldsymbol{\xi}, t) & =\int \eta^{2} f(\boldsymbol{\xi}, \boldsymbol{\eta}, \boldsymbol{x}, t) \mathrm{d} \boldsymbol{\eta} .
\end{aligned}
$$

The conservative macroscopic variables can be computed from these reduced distribution functions as

$$
\rho=\int g \mathrm{~d} \boldsymbol{\xi}, \quad \rho \boldsymbol{U}=\int \boldsymbol{\xi} g \mathrm{~d} \boldsymbol{\xi}, \quad \rho E=\frac{1}{2} \int\left(\xi^{2} g+h\right) \mathrm{d} \boldsymbol{\xi},
$$

and the heat flux can be computed as

$$
\boldsymbol{q}=\frac{1}{2} \int \boldsymbol{c}\left(c^{2} g+h\right) \mathrm{d} \boldsymbol{\xi} .
$$

The evolution equations for the two distribution functions can be deduced from Eq. (1) as

$$
\begin{aligned}
\frac{\partial g}{\partial t}+\boldsymbol{\xi} \cdot \nabla g & =\Omega_{h}=-\frac{1}{\tau}\left[g-g^{S}\right], \\
\frac{\partial h}{\partial t}+\boldsymbol{\xi} \cdot \nabla h & =\Omega_{g}=-\frac{1}{\tau}\left[h-h^{S}\right],
\end{aligned}
$$

where the reduced equilibrium distribution functions $g^{S}$ and $h^{S}$ can be deduced as well,

$$
\begin{aligned}
& g^{S}(\boldsymbol{x}, \boldsymbol{\xi}, t)=\int f^{S}(\boldsymbol{\xi}, \boldsymbol{\eta}, \boldsymbol{x}, t) \mathrm{d} \boldsymbol{\eta}=g^{e q}+g_{\operatorname{Pr}}, \\
& h^{S}(\boldsymbol{x}, \boldsymbol{\xi}, t)=\int \eta^{2} f^{S}(\boldsymbol{\xi}, \boldsymbol{\eta}, \boldsymbol{x}, t) \mathrm{d} \boldsymbol{\eta}=h^{e q}+h_{\operatorname{Pr}},
\end{aligned}
$$


with

$$
\begin{aligned}
g^{e q} & =\frac{\rho}{(2 \pi R T)^{D / 2}} \exp \left[-\frac{c^{2}}{2 R T}\right], \\
h^{e q} & =(3-D) R T g^{e q} \\
g_{\operatorname{Pr}} & =(1-\operatorname{Pr}) \frac{\boldsymbol{c} \cdot \boldsymbol{q}}{5 p R T}\left[\frac{c^{2}}{R T}-D-2\right] g^{e q}, \\
h_{\mathrm{Pr}} & =(1-\operatorname{Pr}) \frac{\boldsymbol{c} \cdot \boldsymbol{q}}{5 p R T}\left[\left(\frac{c^{2}}{R T}-D\right)(3-D)\right] R T g^{e q} .
\end{aligned}
$$

\subsection{Discrete unified gas kinetic scheme on unstructured meshes}

\subsubsection{Updating of the cell-averaged distribution function}

The updating rules for $g$ and $h$ in Eq. (12) have the same structure as

$$
\frac{\partial \phi}{\partial t}+\boldsymbol{\xi} \cdot \nabla \phi=\Omega=-\frac{1}{\tau}\left[\phi-\phi^{S}\right]
$$

for $\phi=g$ or $h$. The generic symbol $\phi$ will be used to denote $g$ and $h$ in the following. The DUGKS is an explicit finite volume scheme for solving Eq. (15). The computation domain is firstly divided into some control volumes (cells). By integrating Eq. 15 in each cell from time $t_{n}$ to $t_{n+1}$, we have

$$
\phi_{j}^{n+1}(\boldsymbol{\xi})-\phi_{j}^{n}(\boldsymbol{\xi})+\frac{\Delta t}{\left|V_{j}\right|} \mathcal{F}_{j}^{n+1 / 2}(\boldsymbol{\xi})=\frac{\Delta t}{2}\left[\Omega_{j}^{n+1}+\Omega_{j}^{n}\right] .
$$

Here $\phi_{j}$ and $\Omega_{j}$ are the cell averaged values of $\phi$ and $\Omega$ in cell $j ;\left|V_{j}\right|$ is the cell's volume and $\Delta t=t_{n+1}-t_{n}$ is the time step. Note that the trapezoidal and middle-point rules are used for the collision and convection terms in Eq. (16), respectively. The term $\mathcal{F}_{j}^{n+1 / 2}$ in Eq. (16) is the flux of $\phi$ across the interface of cell $j$ and is evaluated as

$$
\mathcal{F}_{j}^{n+1 / 2}(\boldsymbol{\xi})=\sum_{k} \boldsymbol{\xi} \cdot \boldsymbol{S}_{j}^{k} \phi\left(\boldsymbol{x}_{j}^{k}, \boldsymbol{\xi}, t_{n+1 / 2}\right)
$$

where $\boldsymbol{S}_{j}^{k}$ is the outward normal vector of the $k$ th face of cell $j$ with face area $\left|\boldsymbol{S}_{j}^{k}\right|$, and $\boldsymbol{x}_{j}^{k}$ is the center of the face. Equation 16 can be rewritten in an explicit form by introducing two new distribution functions [19, 20],

$$
\tilde{\phi}_{j}^{n+1}=\tilde{\phi}_{j}^{+, n}+\mathcal{F}_{j}^{n+1 / 2}
$$


where

$$
\begin{aligned}
\tilde{\phi} & =\phi-\frac{\Delta t}{2} \Omega=\frac{2 \tau+\Delta t}{2 \tau} \phi-\frac{\Delta t}{2 \tau} \phi^{S}, \\
\tilde{\phi}^{+} & =\phi+\frac{\Delta t}{2} \Omega=\frac{2 \tau-\Delta t}{2 \tau+\Delta t} \tilde{\phi}+\frac{2 \Delta t}{2 \tau+\Delta t} \phi^{S} .
\end{aligned}
$$

Due to the conservative property of the collision term, the conservative variables can also be calculated from $\tilde{\phi}$ as 20 .

$$
\rho=\int \tilde{g} \mathrm{~d} \boldsymbol{\xi}, \quad \rho \boldsymbol{U}=\int \boldsymbol{\xi} \tilde{g} \mathrm{~d} \boldsymbol{\xi}, \quad \rho E=\frac{1}{2} \int\left(\xi^{2} \tilde{g}+\tilde{h}\right) \mathrm{d} \boldsymbol{\xi},
$$

and

$$
\boldsymbol{q}=\frac{2 \tau}{2 \tau+\Delta t \operatorname{Pr}} \tilde{\boldsymbol{q}}, \text { with } \tilde{\boldsymbol{q}}=\frac{1}{2} \int \boldsymbol{c}\left(c^{2} \tilde{g}+\tilde{h}\right) \mathrm{d} \boldsymbol{\xi} .
$$

90 Therefore, in actual implementations, we track the evolution of $\tilde{\phi}$ according to Eq. [18), instead of the original distribution function $\phi$ in order to avoid implicit computations. This is also one of the major differences between the DUGKS and the UGKS.

\subsubsection{Flux evaluation on unstructured meshes}

To update $\tilde{\phi}_{j}$ according to Eq. [18), the flux $\mathcal{F}_{j}^{n+1 / 2}$ is required. From the definition of $\mathcal{F}_{j}^{n+1 / 2}$ given by Eq. (17), the original distribution functions at the middle time step at cell interfaces, i.e. $\phi^{n+1 / 2}\left(\boldsymbol{x}_{j}^{k}, \boldsymbol{\xi}\right)$, should be computed. This is done by integrating Eq. 15) from time $t_{n}$ to $t_{n+1 / 2}$ along the characteristic line which ends at the face center $\boldsymbol{x}_{f}$,

$$
\phi^{n+1 / 2}\left(\boldsymbol{x}_{f}, \boldsymbol{\xi}\right)-\phi^{n}\left(\boldsymbol{x}_{f}-\boldsymbol{\xi} s, \boldsymbol{\xi}\right)=\frac{s}{2}\left[\Omega^{n+1 / 2}\left(\boldsymbol{x}_{f}, \boldsymbol{\xi}\right)+\Omega^{n}\left(\boldsymbol{x}_{f}-\boldsymbol{\xi} s, \boldsymbol{\xi}\right)\right],
$$

where $s=t_{n+1 / 2}-t_{n}$ is the half time step. Here the trapezoidal rule is used again for the collision term. Similar to the treatment of Eq. (16), another two auxiliary distribution functions are introduced

$$
\begin{aligned}
\bar{\phi} & =\phi-\frac{s}{2} \Omega=\frac{2 \tau+s}{2 \tau} \phi-\frac{2}{2 \tau} \phi^{S}, \\
\bar{\phi}^{+} & =\phi+\frac{s}{2} \Omega=\frac{2 \tau-s}{2 \tau+s} \bar{\phi}-\frac{2 s}{2 \tau+s} \phi^{S} .
\end{aligned}
$$


Then Eq. (22) can be expressed explicitly as

$$
\bar{\phi}^{n+1 / 2}\left(\boldsymbol{x}_{f}, \boldsymbol{\xi}\right)=\bar{\phi}^{+, n}\left(\boldsymbol{x}_{f}-\boldsymbol{\xi} s, \boldsymbol{\xi}\right) .
$$

In this work, piecewise linear reconstructions in the upstream neighboring cells are employed to interpolate $\bar{\phi}^{+, n}\left(\boldsymbol{x}_{f}-\boldsymbol{\xi} s\right)$ from the cell centered $\bar{\phi}^{+, n}$. The neighboring cells are identified by the direction of the particle velocity $\boldsymbol{\xi}$. To demonstrate this procedure, here we consider a general case as illustrated in Fig. 1, where $A B$ is a cell interface with the center $\boldsymbol{x}_{f}$ and its unit normal vector $\boldsymbol{n}_{f}$ points from cell $P$ to cell $N$. The distribution function $\bar{\phi}^{+}\left(\boldsymbol{x}_{f}-\boldsymbol{\xi} s, \boldsymbol{\xi}, t_{n}\right)$ is

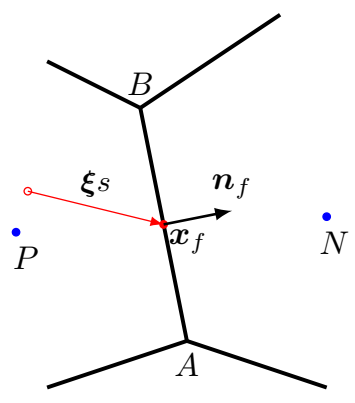

Figure 1: Sketch of two neighboring cells on a general unstructured mesh

evaluated as

$$
\bar{\phi}^{+}\left(\boldsymbol{x}_{f}-\boldsymbol{\xi} s, \boldsymbol{\xi}\right)=\bar{\phi}^{+}\left(\boldsymbol{x}_{C}, \boldsymbol{\xi}\right)+\left(\boldsymbol{x}_{f}-\boldsymbol{x}_{C}-\boldsymbol{\xi} s\right) \cdot \mathcal{L}\left[\bar{\phi}^{+}(\boldsymbol{x}, \boldsymbol{\xi}), \boldsymbol{x}_{C}\right] \nabla \bar{\phi}^{+}\left(\boldsymbol{x}_{C}, \boldsymbol{\xi}\right),
$$

where $C$ stands for $P$ if $\boldsymbol{\xi} \cdot \boldsymbol{n}_{f}>0$, or $N$ otherwise. The gradient $\nabla \bar{\phi}^{+}$at the cell center is calculated using the least square method. For instance, the gradient of cell $P$ is evaluated as

$$
\left(\nabla \bar{\phi}^{+}\right)_{P}=\sum_{i} \omega_{i}^{2} \boldsymbol{G}^{-1} \cdot \boldsymbol{d}_{i}\left[\left(\bar{\phi}^{+}\right)_{P}-\left(\bar{\phi}^{+}\right)_{N_{i}}\right],
$$

where the tensor $\boldsymbol{G}$ is defined as

$$
\boldsymbol{G}=\sum_{i} \omega_{i}^{2} \boldsymbol{d}_{i} \boldsymbol{d}_{i}
$$

95

with $\boldsymbol{d}_{i}$ being the spatial vector from $P$ to its $i$ th adjacent cell center $N_{i}$, and $\omega_{i}=1 /\left|d_{i}\right|$ being the weighting factor. The function $\mathcal{L}\left[\bar{\phi}^{+}(\boldsymbol{x}, \boldsymbol{\xi}), \boldsymbol{x}_{C}\right]$ in Eq. 25] 
denotes the gradient limiter which is used to suppress numerical oscillations in regions with large discontinuities, such as the shock layer in continuum flow regime. In this work, we adopt the Venkatakrishnan limiter [26] which is a typical choice for flow computations on unstructured meshes.

The time step in the DUGKS is determined by the Courant-Friedrichs-Lewy (CFL) condition,

$$
\Delta t=\alpha\left(\frac{\Delta x}{|\boldsymbol{U}|+|\boldsymbol{\xi}|}\right)_{\min },
$$

where $0<\alpha<1$ is the CFL number and $\Delta x$ is the distance between the centers of two neighboring cells that share an interface. It should be noted that the CFL condition is imposed by the treatment of the convection term in the governing equation. Usually the collision term also poses a restriction on the time step since the computation can be unstable if $\Delta t$ is much larger than the mean collision time $\tau$. However, in DUGKS, as long as the computation is stable, accurate solutions can be obtained even if $\Delta t$ is much larger than the mean collision time [20, due to coupled treatment of the collision and transport in the construction of numerical flux. While in many DVM schemes, the lack of collision contribution in the numerical flux leads to a numerical viscosity proportional to the time step [13, 27], such that the time step must be smaller than the mean collision time in order to keep the numerical viscosity being much smaller than the physical viscosity.

After getting $\bar{\phi}$ at face centers according to Eqs. (24) and (25), the original distribution functions $\phi$ can be recovered from Eq. (23a). The macro variables at time $t_{n+1 / 2}$ used to evaluate the equilibrium distribution functions $\phi^{S}$ are calculated from $\bar{\phi}$ as

$$
\rho=\int \bar{g} d \boldsymbol{\xi}, \quad \rho \boldsymbol{U}=\int \boldsymbol{\xi} \bar{g} d \boldsymbol{\xi}, \quad \rho E=\frac{1}{2} \int\left(\xi^{2} \bar{g}+\bar{h}\right) d \boldsymbol{\xi},
$$

and

$$
\boldsymbol{q}=\frac{2 \tau}{2 \tau+s \operatorname{Pr}} \overline{\boldsymbol{q}}, \text { with } \overline{\boldsymbol{q}}=\frac{1}{2} \int \boldsymbol{c}\left(c^{2} \bar{g}+\bar{h}\right) d \boldsymbol{\xi} .
$$

Then the flux across each cell interface can be evaluated according to Eq. (17).

${ }_{115}$ Finally, the cell centered $\tilde{\phi}$ can be advanced to the new time level according to Eq. 18. 
The updating procedures presented above are all based on continuous velocity space for convenience. In actual implementations, the continuous velocity space is discretized into a finite discrete velocity set $\left\{\boldsymbol{\xi}_{i}\right\}$ like the DVM [25], and the distribution functions such as $\tilde{g}$ and $\tilde{h}$ are defined at these discrete velocity points as $\tilde{g}_{i}$ and $\tilde{h}_{i}$. Proper quadrature rules such as the Newton-Cotes quadrature or the Gauss-Hermite quadrature are then used to approximate the moments,

$$
\rho=\sum_{i} \varpi_{i} \tilde{g}_{i}, \quad \rho \boldsymbol{U}=\sum_{i} \varpi_{i} \boldsymbol{\xi}_{i} \tilde{g}_{i}, \quad \rho E=\frac{1}{2} \sum_{i} \varpi_{i}\left[\xi_{i}^{2} \tilde{g}_{i}+\tilde{h}_{i}\right]
$$

where $\varpi_{i}$ are the weight coefficients.

\section{Numerical examples}

We apply the proposed DUGKS on unstructured meshes to both internal and external flows to demonstrate its performance in multiscale flow simulations. The first one is the two dimensional lid driven cavity flow in different regimes, the second one is a multiscale unsteady gas expansion problem in which the Knudsen number ranges from $10^{-3}$ to 10 , the third one is a supersonic rarefied gas flow with Mach number $\mathrm{Ma}=5$ passing through a circular cylinder at $\mathrm{Kn}=0.1$ and

1 , the forth one is a Mach 2 rarefied gas flow passing through a two side-by-side circular cylinders at $\mathrm{Kn}=0.1$, the last one is the impulsive start problem, which is used to verify the uniform convergence rate of the DUGKS.

The simulations start from equilibrium states based on given initial flow variables. For a steady problem, the flow field gradually evolves into the final steady state. The flow field will be assumed to be steady when the average relative change of the temperature field in a two-successive steps is less than $10^{-8}$, i.e.,

$$
\varepsilon^{n}=\frac{\sum_{i}\left|T_{i}^{n+1}-T_{i}^{n}\right|}{\sum_{i} T_{i}^{n}}<10^{-8}
$$

where the summations are taken over all cells.

In all tests, the gas is argon with molecular mass $m=6.63 \times 10^{-26} \mathrm{~kg}$ and the molecular diameter $d=4.17 \times 10^{-10} \mathrm{~m}$. The exponent $\omega$ in the viscosity- 
temperature relation is 0.81 , corresponding to the variable hard sphere (VHS) model of molecular interaction [1. However, in the calculation of the reference viscosity by Eq. 4. $\omega$ is 0.5 , corresponding to the hard sphere (HS) model of molecular interaction [1].

\subsection{Lid driven cavity flow}

The two dimensional lid driven cavity flow is a standard benchmark problem for validating classical CFD methods in continuum regime. This problem has also been studied recently by Benzi et al. 28] using a parallel DSMC code at Knudsen numbers $\mathrm{Kn}=10,1.0,0.075$, and becomes a benchmark test to validate different schemes in the whole flow regimes [17, 13, 19, 21]. To demonstrate the capability of the present DUGKS on unstructured meshes, we also simulate this flow in different regimes.

The flow domain is a square cavity with length $L=1 \mathrm{~m}$. The upper wall moves with a constant velocity $U_{\mathrm{w}}$, while other walls are kept fixed. The temperature at the four walls is fixed at $T_{\mathrm{w}}=273 \mathrm{~K}$ and is used as the reference temperature. The walls are fully diffusive and the boundary conditions are the same as that presented in Refs [19, 20]. The Knudsen number is defined as the ratio of the mean free path corresponding to the initial density $\rho_{\text {ref }}$ and the cavity length $L$. Different Knudsen numbers can be obtained by adjusting the initial density.

Both rarefied and continuum flows are simulated. In rarefied regimes, three values of the Knudsen number, $\mathrm{Kn}=10,1$ and 0.075 , are considered. The velocity of the upper wall is set to be $U_{\mathrm{w}}=50 \mathrm{~m} / \mathrm{s}$, which is the same as that used in the DSMC and the UGKS simulations [28, 17]. The corresponding Knudsen numbers are $3.7763 \times 10^{-4}$ and $1.5105 \times 10^{-4}$, respectively. Furthermore, the Mach number is $\mathrm{Ma}=0.1$, so that the flow is nearly incompressible and the solutions in the continuum regime can be compared with the benchmark solutions [29] of the incompressible Navier-Stokes equations.

In the previous work [19, the DUGKS with structured meshes has been 


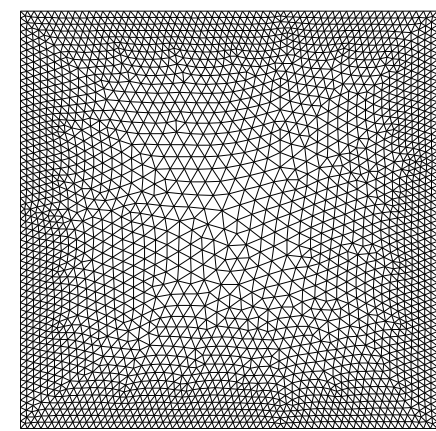

(a)

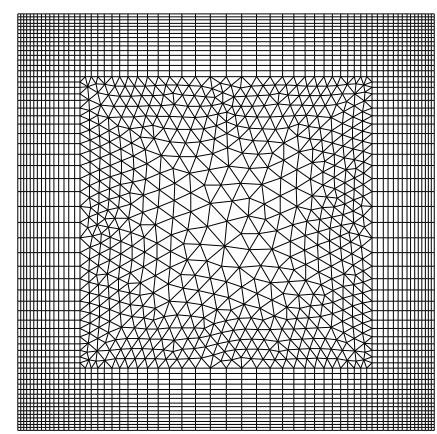

(b)

Figure 2: Meshes for the cavity flow. (a) Kn=10,1 and 0.075. (b) $\mathrm{Re}=400$ and 1000.

unstructured meshes to demonstrate the performance of the proposed method. Figure 2 presents the meshes used for flows with different Knudsen numbers and Reynolds numbers. Note that the mesh in Fig. 2(b) is a hybrid mesh with quadrilateral cells near the walls. It is suitable to capture the boundary layer effect which is important for continuum flows.

The discretization of velocity space and quadrature rules used depend on the Knudsen number. For highly rarefied flows (i.e. $\mathrm{Kn}=10,1$ ), we use the NewtonCotes rule with $101 \times 101$ velocity points distributed uniformly in the range of $\left[-4 \sqrt{2 R T_{\mathrm{w}}}, 4 \sqrt{2 R T_{\mathrm{w}}}\right] \times\left[-4 \sqrt{2 R T_{\mathrm{w}}}, 4 \sqrt{2 R T_{\mathrm{w}}}\right]$. For the case of $\mathrm{Kn}=0.075$, we adopt the half-range Gauss-Hermit quadrature with $28 \times 28$ velocity points. For continuum flows, we employ the half-range Gauss-Hermit quadrature rule with $8 \times 8$ velocity points. The CFL number is fixed at 0.8 in all simulations unless stated otherwise.

Figures 345 present the temperature field, heat flux, and velocity $(U, V)$ on the vertical and horizontal center lines, for the cases of $\mathrm{Kn}=10,1$ and 0.075 , respectively, together with the DSMC solutions. It can be seen that the present results agree well with DSMC data. It is interesting to note that the direction of the heat flux is not following temperature gradient in each case, which indicates the breakdown of Fourier law at the Knudsen number 0.075 and even smaller ones. 


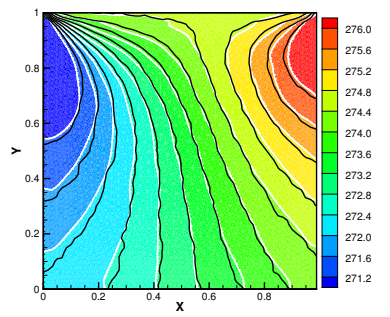

(a)

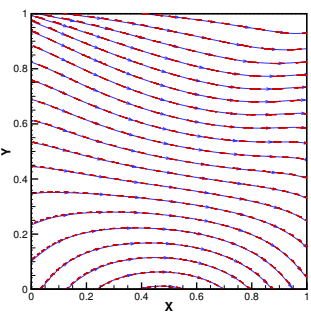

(b)

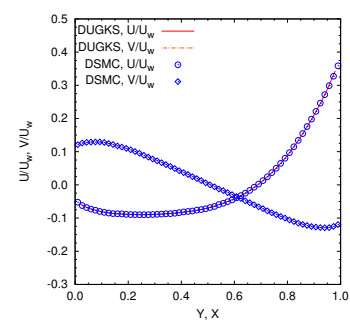

(c)

Figure 3: Results of the cavity flow at $\mathrm{Kn}=10$. (a) Temperature contours, black line: DSMC; white line with background: DUGKS. (b) Heat flux: blue solid line with arrows: DSMC; red dashed line : DUGKS. (c) U-velocity along vertical center line and V-velocity along horizontal central line.

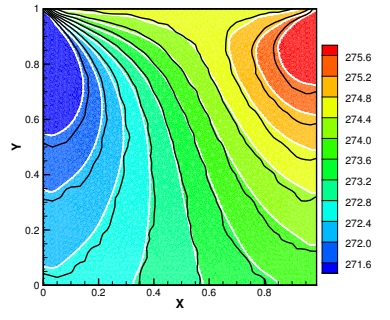

(a)

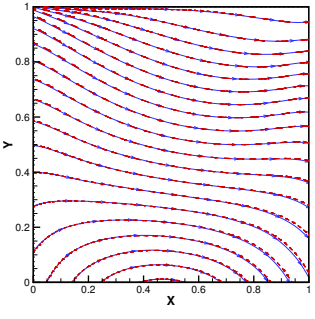

(b)

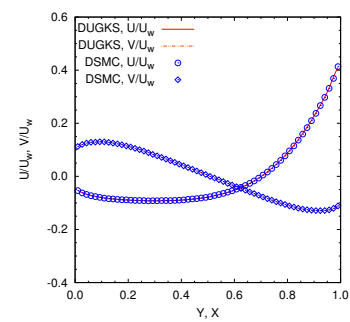

(c)

Figure 4: Results of the cavity flow at $\mathrm{Kn}=1$. (a) Temperature contours, black line: DSMC; white line with background: DUGKS. (b) Heat flux: blue solid line with arrows: DSMC, red dashed line : DUGKS. (c) U-velocity along vertical center line and V-velocity along horizontal central line. 


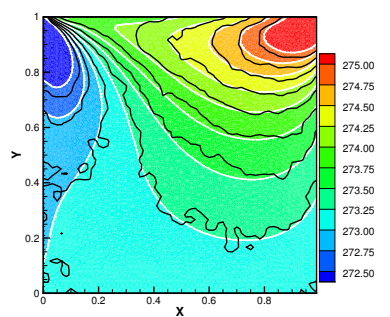

(a)

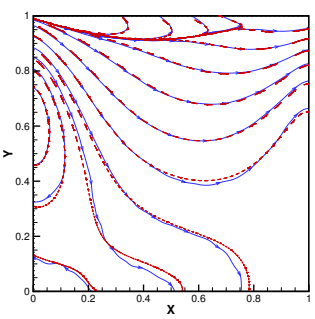

(b)

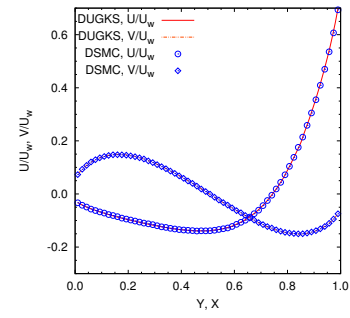

(c)

Figure 5: Results of the cavity flow at $\mathrm{Kn}=0.075$. (a) Temperature contours, black line: DSMC; white line with background: DUGKS. (b) Heat flux: blue solid line with arrows: DSMC; red dashed line : DUGKS. (c) U-velocity along vertical center line and V-velocity along horizontal central line.

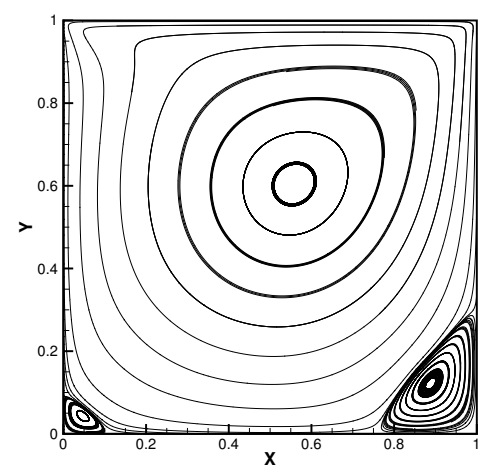

(a)

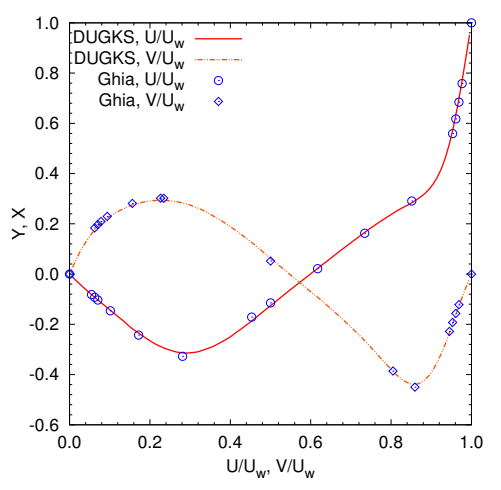

(b)

Figure 6: Results of the cavity flow at $\mathrm{Re}=400, \mathrm{Kn}=3.7763 \times 10^{-4}$. (a) Velocity streamline (b) U-velocity alone vertical central line and $\mathrm{V}$-velocity alone horizontal central line. 


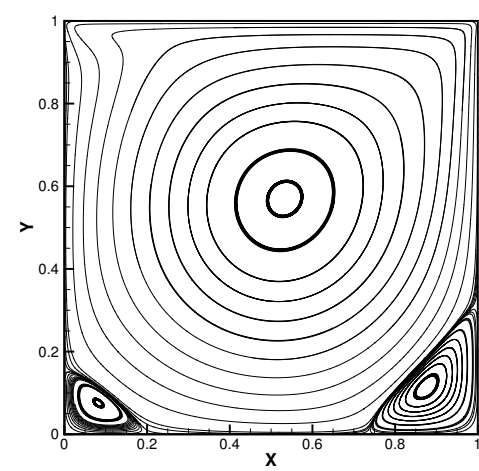

(a)

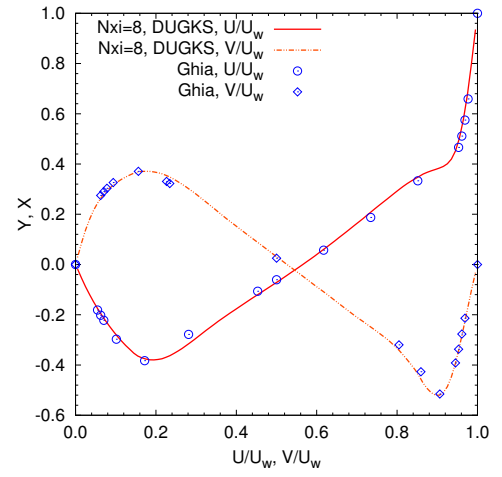

(b)

Figure 7: Results of the cavity flow at $\mathrm{Re}=1000, \mathrm{Kn}=1.5105 \times 10^{-4}$. (a) Velocity streamline. (b) U-velocity alone vertical central line and V-velocity alone horizontal central line.

Figures 6 and 7 show the streamlines and velocity profiles for the cases of $\operatorname{Re}=400$ and 1000, respectively. The benchmark solutions [29] are also included for comparison. Even with the cell size and time step size being much larger than the mean free path and mean collision time in these cases, for example, at $\operatorname{Re}=1000$, the smallest cell size is about 45 times of the mean free path, and at steady state the time step size is 20 times of the mean collision time, the DUGKS results still have good agreement with the benchmark data. So the DUGKS recovers the Navier-Stokes solutions in the continuum limit. We would also like to point out that for most traditional DVM methods, the numerical dissipation is proportional to cell size due to the splitting treatment of particle transport and collision processes. This may lead to significant errors for unstructured meshes as the cell size changes dramatically. The above results indicate that the DUGKS can avoid this kind of difficulty with coupled treatment of particle transport and collision. The stable computations of the cavity flow in the three different flow regimes with the CFL number fixed at 0.8 also indicate the uniform stability of the current method with respect to the Kn. 


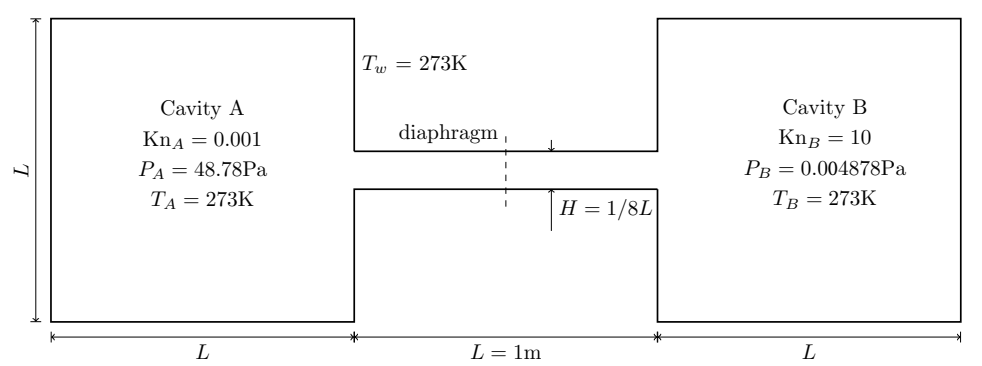

Figure 8: Gas expansion between two cavities connected by a channel.

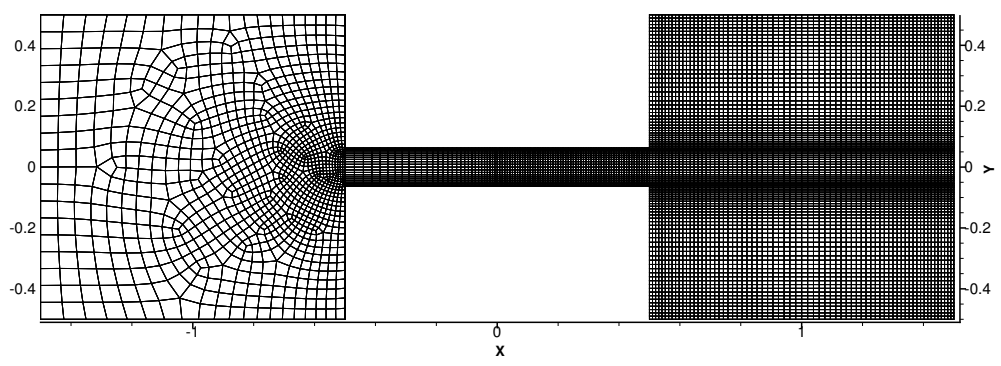

Figure 9: Mesh for the gas expansion case.

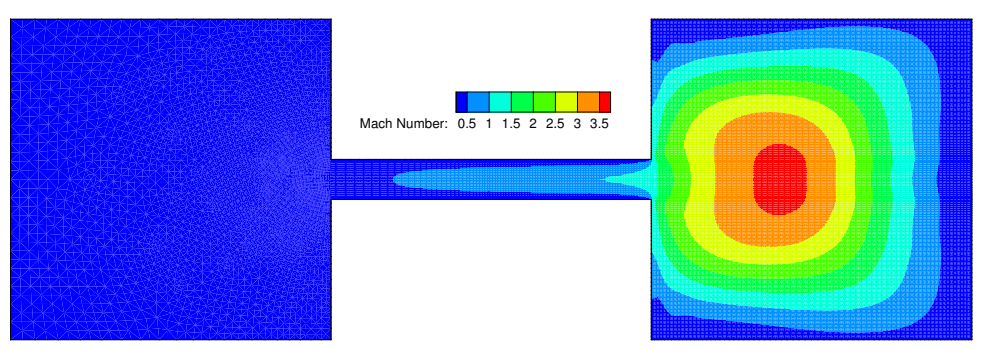

Figure 10: Mach number contours for the gas expansion problem at time $t / t_{c}=1$. 


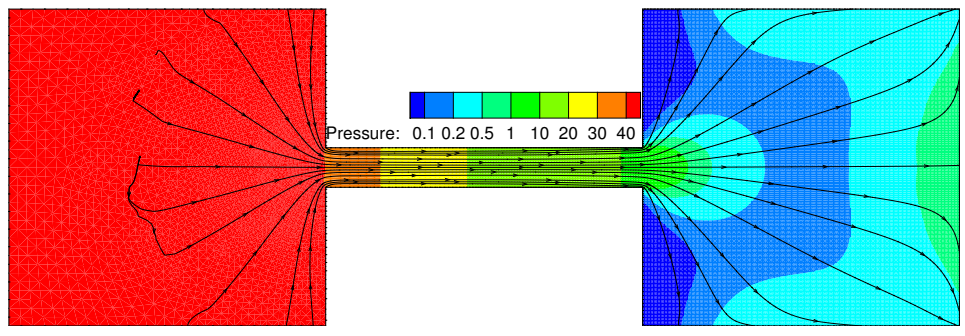

Figure 11: Pressure contours and streamlines for the gas expansion problem at time $t / t_{c}=1$.

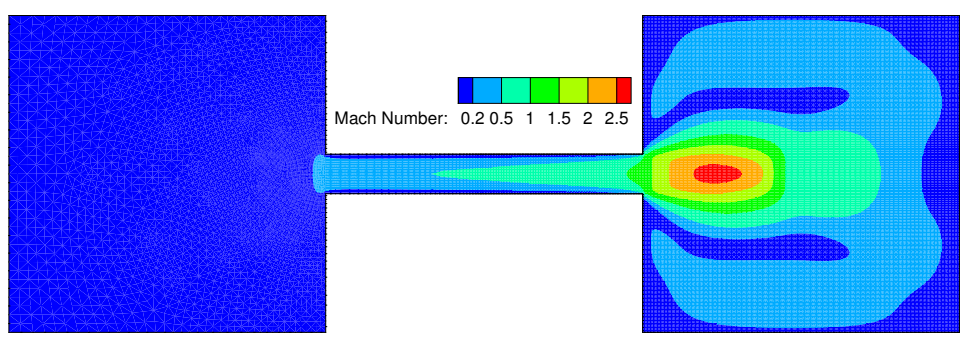

Figure 12: Mach number contours for the gas expansion problem at time $t / t_{c}=4$.

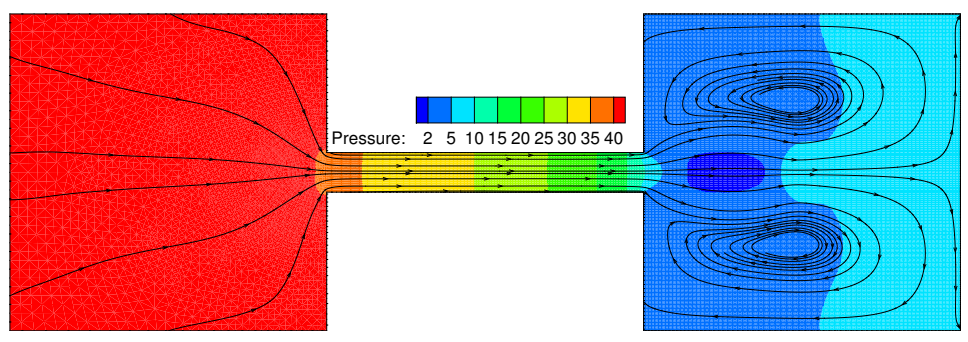

Figure 13: Pressure contours and streamlines for the gas expansion problem at time $t / t_{c}=4$. 


\subsection{Multiscale flow expansion between two connected cavities}

In the above subsection, the cavity flow at specific flow regimes have been simulated. Now we consider a gas expansion between two connected cavities

channel and cavity $B$ at the early stage of expansion. In our simulations, the CFL number is set to be 0.8 . 


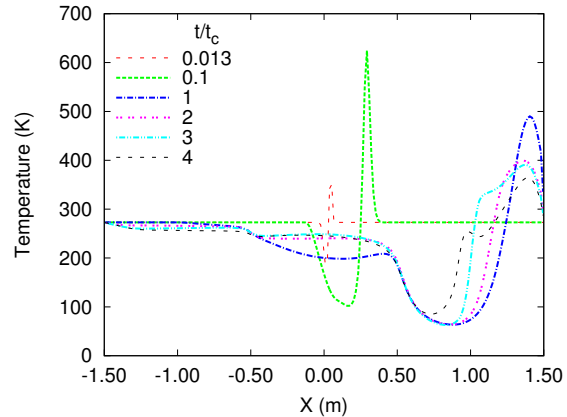

(a)

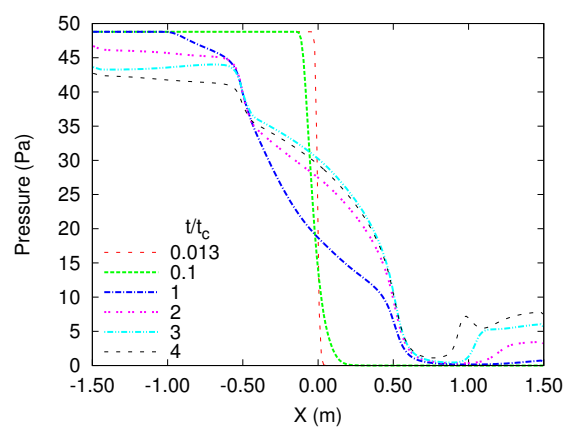

(c)

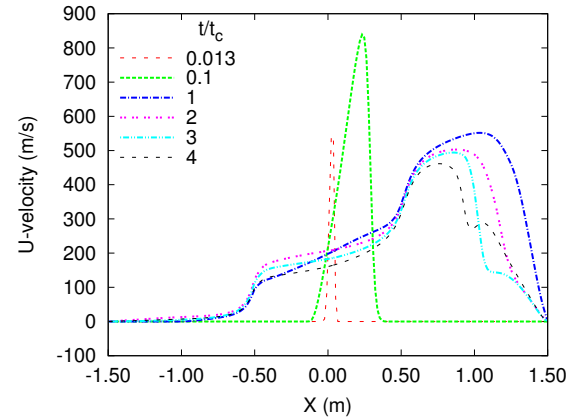

(b)

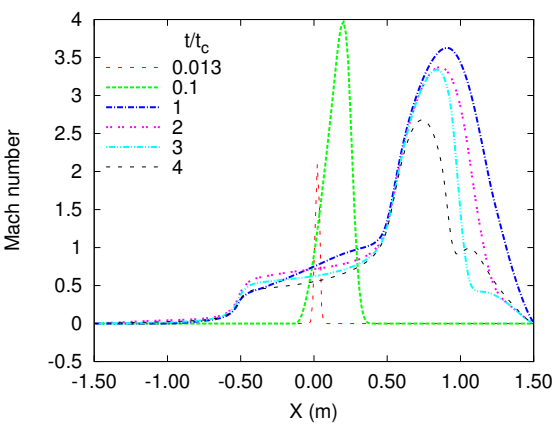

(d)

Figure 14: Temperature (a), horizontal velocity (b), pressure (c) and Mach number (d) along the horizontal center line across the cavities and the channel at different times for the gas expansion problem. 


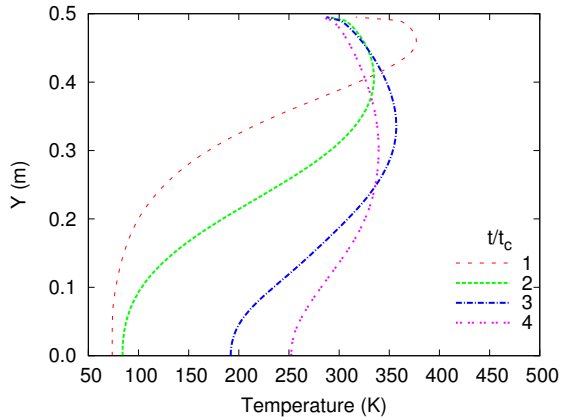

(a)

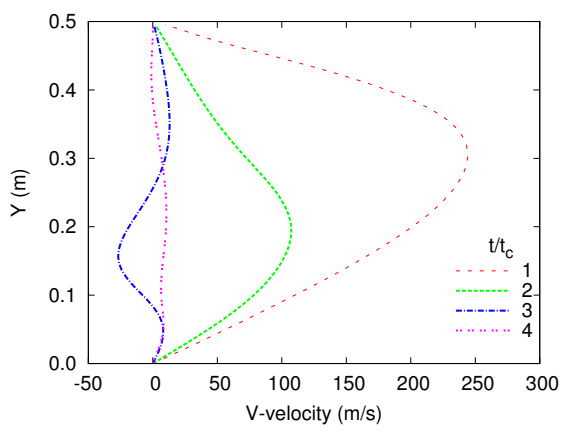

(c)

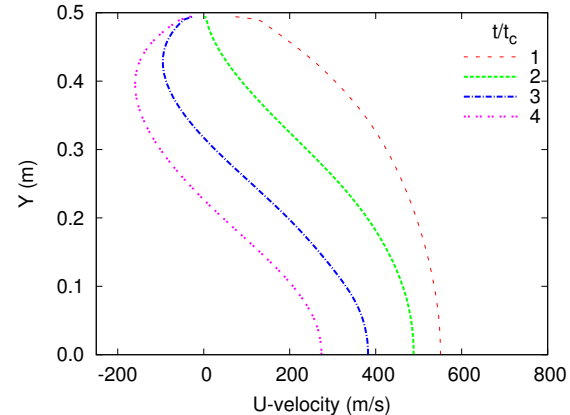

(b)

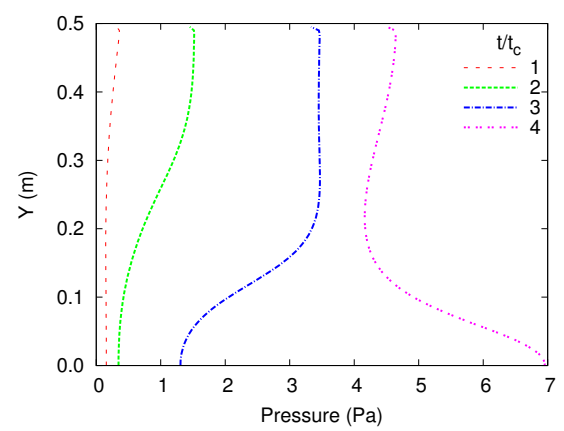

(d)

Figure 15: Temperature (a), horizontal velocity (b), vertical velocity (c) and pressure (d) along the vertical center line (upper half) of the cavity $B$ at different times for the gas expansion problem. 
We can define a characteristic time of the system as $t_{c}=L / \sqrt{2 R T_{\mathrm{w}}}$, and the flow fields at different times are measured. The local Mach number, pressure, and streamlines at times $t / t_{c}=1$ and 4 are presented in Figs. 10.13 . Figs. 10 and 11 show that the shock wave reaches the center of cavity $B$ at time $t / t_{c}=1$. At this moment the gas is still very rarefied there associated with ballistic behavior, and there is no vortex formation. As the gas moves into cavity $B$ continuously, the pressure in cavity $B$ rises with time, but the pressure ratio between the two cavities is high enough to form a supersonic jet at the outlet of the channel. The initial shock wave disappears, and two symmetric vortexes appear in cavity $B$ at a later time.

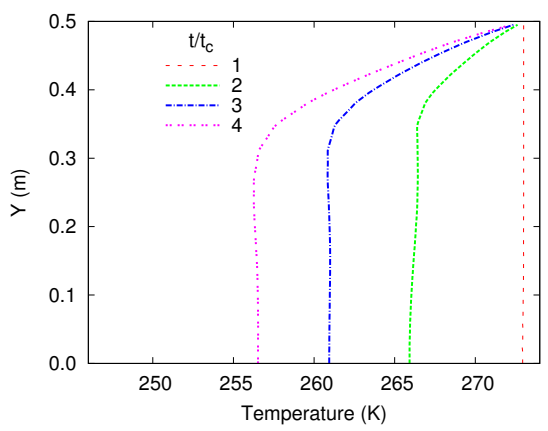

(a)

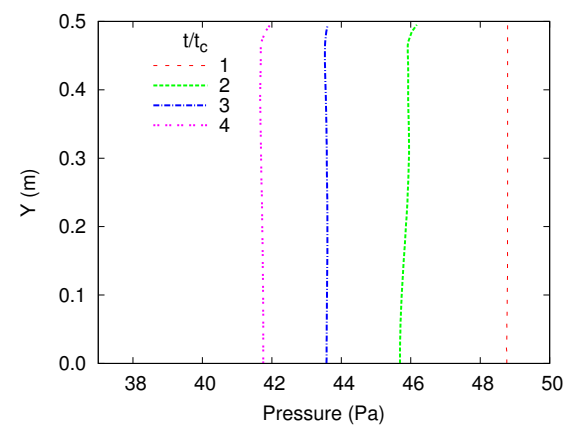

(b)

Figure 16: Temperature (a) and pressure (b) along the vertical center line (upper half) of the cavity $A$ at different times for the gas expansion problem.

To get a detail information of the expansion process, we show the temperature, U-velocity and pressure profiles along the horizontal symmetric line of the system at times $t / t_{c}=0.013,0.1,1,2,3$ and 4 in Fig. 14. It can be seen that at the early stage $\left(t / t_{c}=0.013\right.$ and 0.1$)$, the shock wave propagates in the channel, and the flow variables change sharply across the shock. With time increments, the pressure difference between the two cavities decreases. Consequently, the shock becomes weaker and the flow rate decreases gradually.

To quantify the results, the temperature, velocity, and pressure profiles along the vertical center lines of the two cavities at different times are presented in 
Figs. 15 and 16 respectively. Here only the results at the upper half $(0<y<$ $L / 2$ ) of the domain are shown owing to the symmetry of the flow. Fig. 15(b) shows a counterclockwise vortex development in the upper half of cavity $B$, which enhances heat convection in the gas. Consequently, the temperature field becomes uniform gradually, as indicated in Fig. 15(a). On the other hand, the flow in cavity $A$ changes slightly. Actually, the temperature and pressure are almost uniform at each time. With the decreasing of pressure in the cavity, the temperature reduces as the internal energy is converted to the kinetic energy.

\subsection{Mach 5 rarefied gas flow passing through a circular cylinder}

To further demonstrate the performance of the DUGKS on unstructured meshes for high speed non-equilibrium external flows, we simulate the rarefied gas flow passing through a circular cylinder. This problem has been studied before [16] using the UGKS method with structured mesh. We here adopt the same configuration and parameters as the early simulations. The free-stream Mach number is $\mathrm{Ma}_{\infty}=5$, and the radius of the cylinder is $r=0.01 \mathrm{~m}$. Two Knudsen numbers are considered $\left(\mathrm{Kn}_{\infty}=\lambda_{\infty} / r=0.1\right.$ and 1$)$. The free-stream gas temperature is $T_{\infty}=273 \mathrm{~K}$ and is used as the reference temperature. The surface of the cylinder is maintained at constant temperature of $T_{\mathrm{w}}=273 \mathrm{~K}$, and diffusive boundary condition is assumed. The outer boundary of the computational domain is a circle with a diameter of $D_{o}=22 r$, and forms a concentric annular along with the surface of the cylinder. The distribution functions coming to the computational domain from the outer boundary are equilibrium state with the free-stream flow conditions.

Hybrid meshes are adopted again for this test case (see Fig. 17). Locally refined quadrilateral cells are used near the cylinder to resolve the boundary layer. We note that the mesh resolution in the normal direction of the cylinder wall should be fine enough to capture the large flow gradients correctly in the boundary layer. For the case of $\mathrm{Kn}=0.1$, the mesh spacing around the cylinder wall is finer than that for $\mathrm{Kn}=1$ (see Fig. 17(b)) since the boundary layer become thinner as Kn decreases. The fine meshes around the cylinder wall are 


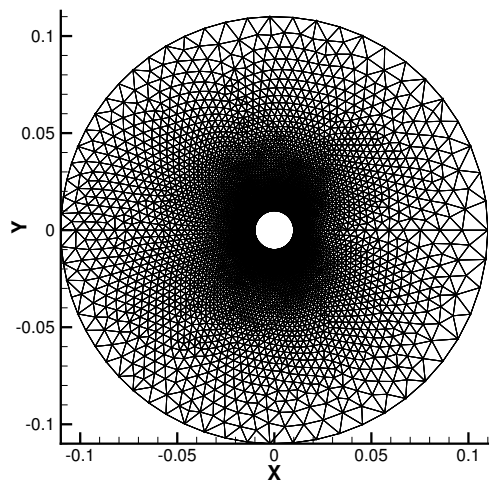

(a)

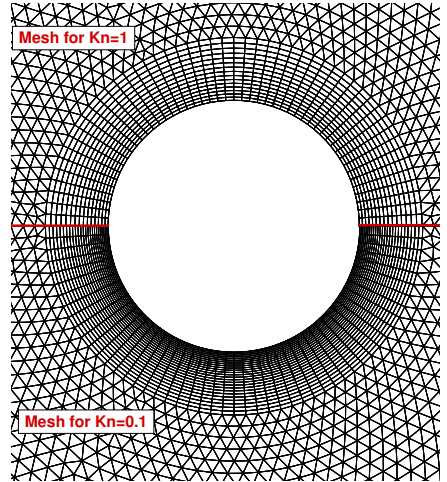

(b)

Figure 17: Meshes for the flow passing through a cylinder. (a) Global view of the mesh. (b) Local view of the meshes around the cylinder surface, upper: $\mathrm{Kn}=1$, lower: $\mathrm{Kn}=0.1$.

only used to capture the large gradients of the flow field, but not to resolve the mean free path scale. Actually, based on the posterior estimation, the mesh spacing around the stagnation point for the case of $\mathrm{Kn}=1$ is about 2 times of the mean free path there.

In our computations, the velocity space is discretized into $89 \times 89$ uniform grid points in the range of $\left[-15 \sqrt{2 R T_{\infty}}, 15 \sqrt{2 R T_{\infty}}\right] \times\left[-15 \sqrt{2 R T_{\infty}}, 15 \sqrt{2 R T_{\infty}}\right]$, and the Newton-Cotes quadrature rule is used for the numerical integration. To validate our simulation results, we use the open source $d s m c F o a m$ solver [30] to obtain the DSMC results under the same flow conditions and computational domain.

The contours of temperature and Mach number for the case of $\mathrm{Kn}=0.1$ are shown in Fig. 18. Also included are the DSMC solutions. The temperature and U-velocity profiles along the stagnation line are shown in Fig. 19 . Clearly both temperature and Mach number distributions of the DUGKS results agree with those of the DSMC results perfectly. However, there are some small discrepancies in the front of the bow shock, which can be seen more clearly in the temperature profile. This is due to the intrinsic defect of the Shakhov model 


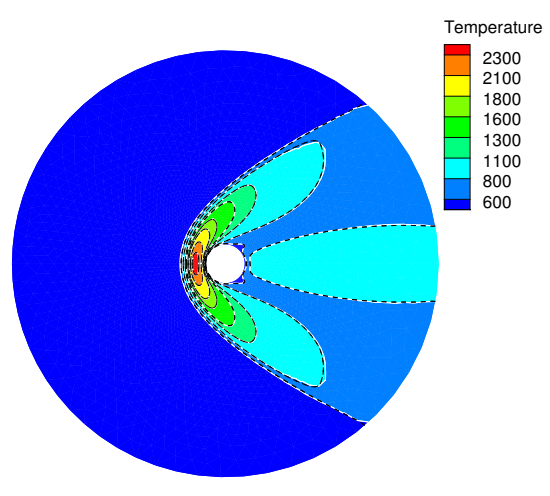

(a)

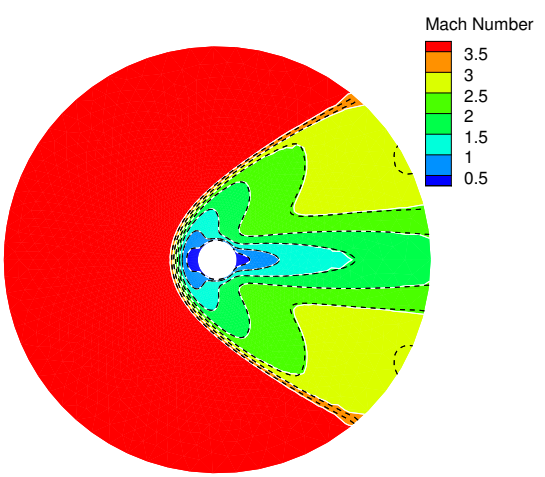

(b)

Figure 18: Temperature (a) and Mach number (b) distribution for the flow passing through a cylinder at $\mathrm{Kn}=0.1$. Solid white line with colored background: DUGKS, dashed black line: DSMC.

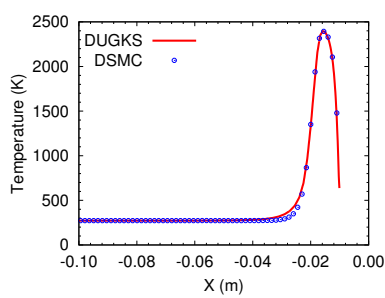

(a)

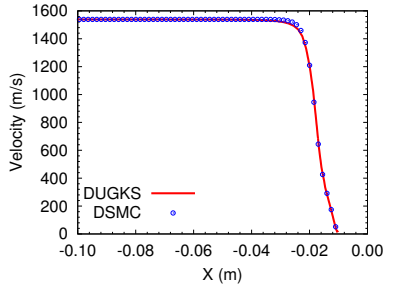

(b)

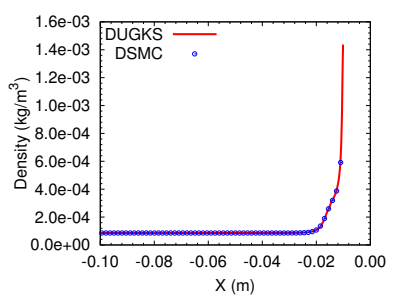

(c)

Figure 19: Temperature (a), velocity (b) and density (c) profiles alone the stagnation line for flow past a cylinder at $\mathrm{Kn}=0.1$.

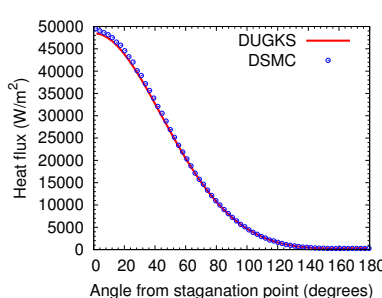

(a)

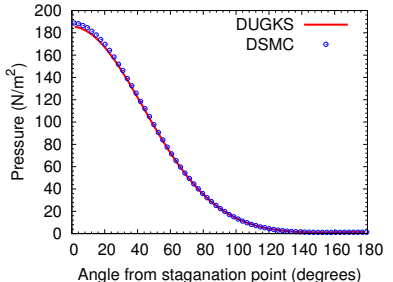

(b)

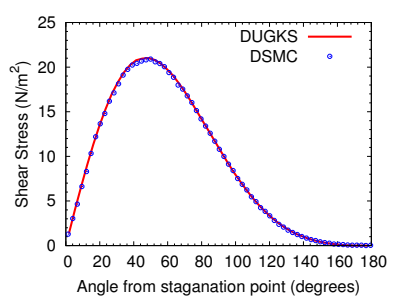

(c)

Figure 20: Heat flux (a), pressure (b) and shear stress (c) alone the surface for the flow passing through a cylinder at $\mathrm{Kn}=0.1$. 


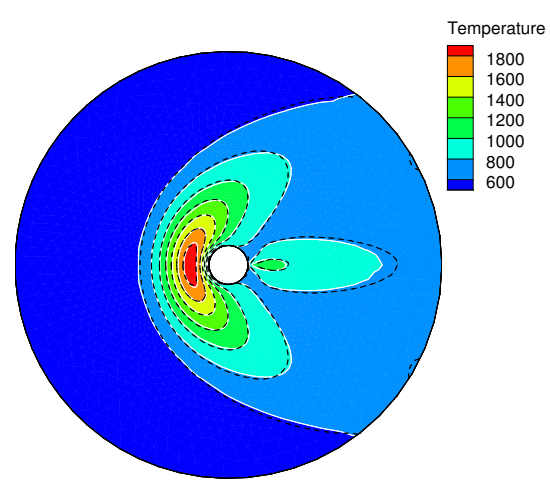

(a)

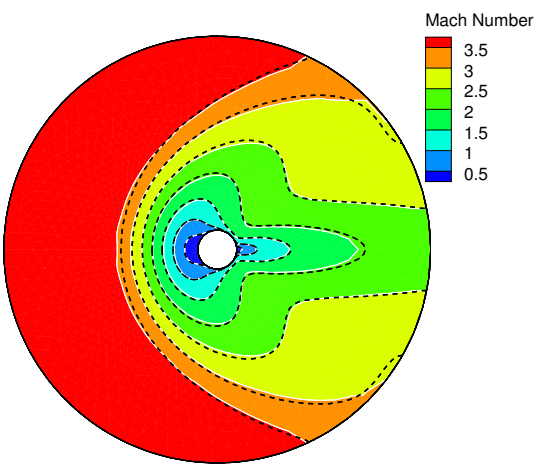

(b)

Figure 21: Temperature and Mach number distributions for the flow passing through a cylinder at $\mathrm{Kn}=1$. Solid white line with colored background: DUGKS, dashed black line: DSMC.

used in the current DUGKS [1], where the collision frequency is independent of particle velocities. Despite of the small deviations, the temperature agrees well with the DSMC results in the downstream the shock. The heat flux, normal pressure and shear stress distribution along the cylinder's surface predicted by the DUGKS and DSMC agree with each other quite well, as shown in Fig. 20.

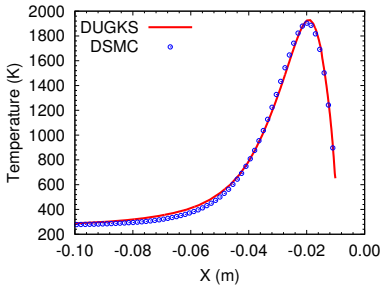

(a)

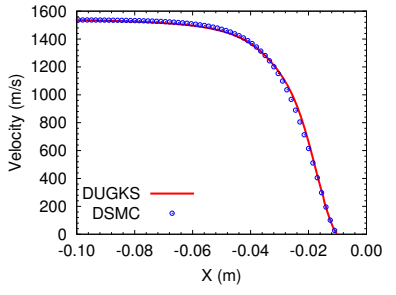

(b)

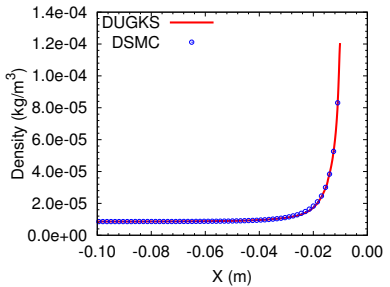

(c)

Figure 22: Temperature (a), velocity (b) and density (c) profiles alone the stagnation line for flow passing through a cylinder at $\mathrm{Kn}=1$.

For the case of $\mathrm{Kn}=1$, the temperature and Mach number distributions are presented in Fig. 21. The temperature, U-velocity, and density profiles along the stagnation line are shown in Fig. 22. These results show that the DUGKS 


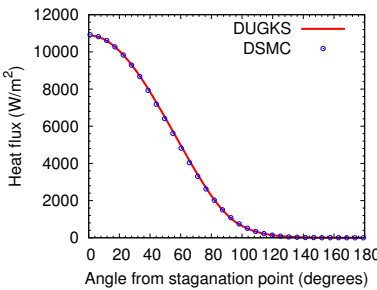

(a)

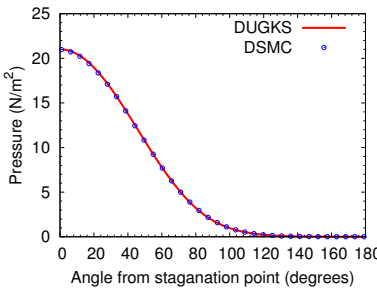

(b)

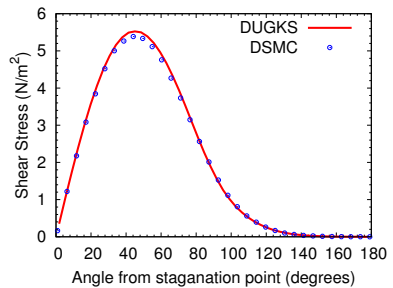

(c)

Figure 23: Heat flux (a), pressure (b) and shear stress (c) alone the surface for the flow passing through a cylinder at $\mathrm{Kn}=1$.

results agree with the DSMC results as well. The discrepancies in the front of the bow shock are slightly more obvious. Due to the increment of Kn, the nonequilibrium effects get stronger, thus the Shakhov model deviates more from the full Boltzmann collision operator. However, the heat flux, normal pressure, and shear stress along surface of cylinder predicted by DUGKS are still quite satisfactory in comparison with the DSMC results, as shown in Fig. 23 . These results demonstrate that although the Shakhov model with intrinsic defects is used in the current numerical modeling, the DUGKS still gives rather satisfactory predictions, particularly the flow behaviors near the body. The DUGKS can be a very useful engineering tool for hypersonic rarefied flow applications, especially in the regime of $\mathrm{Kn}<0.1$.

\subsection{Mach 2 rarefied gas flow passing through two side-by-side circular cylinders}

We further apply the DUGKS to the flow over two circular cylinders in a side-by-side arrangement, as sketched in Fig. 24. The computational domain is a $0.18 \mathrm{~m}$ by $0.2 \mathrm{~m}$ rectangular. Two cylinders with radius of $r=0.01 \mathrm{~m}$ are placed vertically in the computational domain with a gap of $2 r$. The centers of the cylinders are $8 r$ away from the left boundary. The gas is argon and the freestream Mach number and temperature are $\mathrm{Ma}=2$ and $T_{\infty}=273$, respectively. The Knudsen number based on the radius of the cylinders and the mean free path of free-stream gas is 0.1 . For this geometry, it is difficult to use a simple structured mesh to represent the configuration. But for the present DUGKS, we 
can easily setup a hybrid structured-unstructured mesh, as illustrated in Fig. 24 The velocity space is discretized into $49 \times 49$ uniform grid points in the range of $325\left[-6 \sqrt{2 R T_{\infty}}, 6 \sqrt{2 R T_{\infty}}\right] \times\left[-6 \sqrt{2 R T_{\infty}}, 6 \sqrt{2 R T_{\infty}}\right]$, and the Newton-Cotes rule is used for the calculation of the moments. In our simulations, the CFL number is set to be 0.8 .

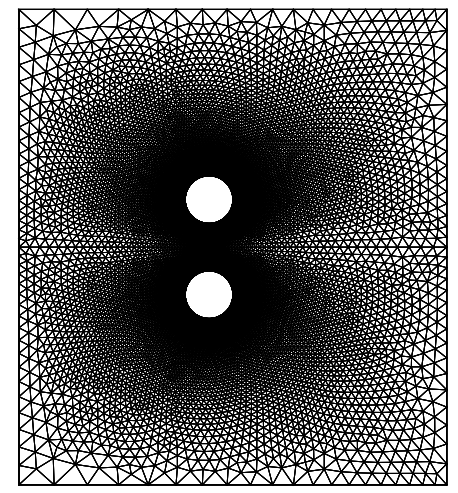

Figure 24: Mesh for the flow past two cylinders case.

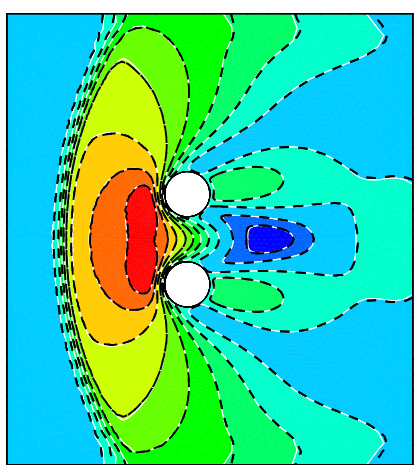

(a)

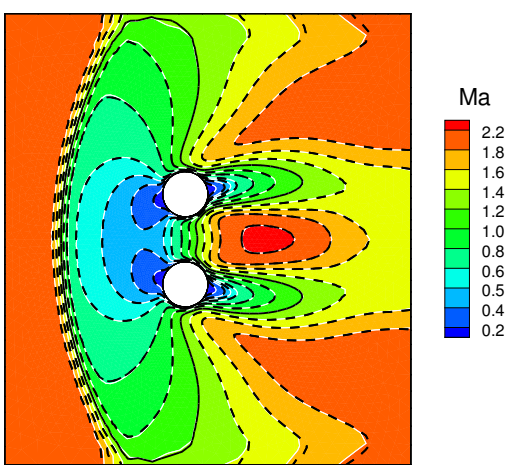

(b)

Figure 25: Temperature (a) and mach number (b) distribution for the flow passing through two cylinders case. Solid white line with colored background: DUGKS; dashed black line: DSMC.

The results of the DUGKS are compared with those of the dsmcFoam solver [30. Fig. 25 shows the temperature and Mach number distributions predicted by the 


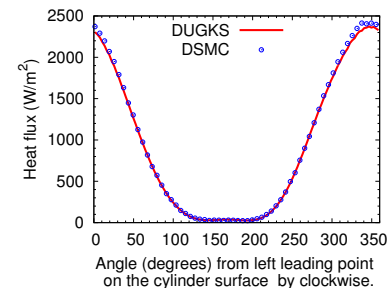

(a)

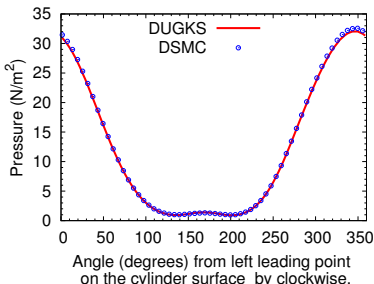

(b)

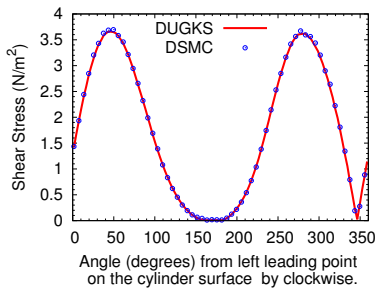

(c)

Figure 26: Heat flux (a), pressure (b) and shear stress (c) alone the surface of the upper cylinder for the flow past tow cylinders case.

\subsection{Impulsive start problem}

In this test, we investigate the spatial convergence order of the DUGKS at varies flow regimes (Knudsen numbers). The problem considered is the flow of argon gas between two infinitely long parallel plates, placed at $y=L / 2$ and $y=$ $-L / 2$. The surfaces of the plates are maintained at uniform temperature $T_{\mathrm{w}}$ and are assumed to be diffusive boundaries. The flow filed is initially set at uniform temperature $T_{w}$, density $\rho_{0}$ and is static. At time $t=0$, both of the plates 
start to move in the $X$ direction impulsively with velocity $U_{\mathrm{w}}=0.15 \sqrt{2 R T_{\mathrm{w}}}$. The Knudsen number Kn is based on the distance between the plates $L$ and the initial density $\rho_{0}$. Three $\mathrm{Kn}$ values, $\mathrm{Kn}=10,0.1$ and 0.001 are considered. The characteristic time of the system is chosen as $t_{c}=L / \sqrt{2 R T_{\mathrm{w}}}$.

Table 1: $L_{2}$ errors of the velocity field and convergence orders at varies Knudsen numbers for the impulsive start problem

\begin{tabular}{rrrrrrrrr}
\hline & $\mathrm{Kn}=10$ & & & $\mathrm{Kn}=0.1$ & \multicolumn{3}{c}{$\mathrm{Kn}=0.001$} \\
\hline$N_{i}$ & $\|\delta u\|_{2}^{i}$ & $E_{i}^{i+1}$ & $N_{i}$ & $\|\delta u\|_{2}^{i}$ & $E_{i}^{i+1}$ & $N_{i}$ & $\|\delta u\|_{2}^{i}$ & $E_{i}^{i+1}$ \\
\hline 5 & $6.05 \mathrm{E}-02$ & & 10 & $4.46 \mathrm{E}-03$ & & 20 & $7.82 \mathrm{E}-02$ & \\
10 & $6.10 \mathrm{E}-03$ & 3.31 & 20 & $1.19 \mathrm{E}-03$ & 1.90 & 40 & $2.07 \mathrm{E}-02$ & 1.92 \\
20 & $1.30 \mathrm{E}-03$ & 2.22 & 40 & $3.18 \mathrm{E}-04$ & 1.91 & 80 & $3.30 \mathrm{E}-03$ & 2.65 \\
40 & $3.15 \mathrm{E}-04$ & 2.04 & 80 & $8.16 \mathrm{E}-05$ & 1.96 & 160 & $4.91 \mathrm{E}-04$ & 2.75 \\
\hline$n$ & & 2.50 & & & 1.92 & & & 2.46 \\
\hline
\end{tabular}

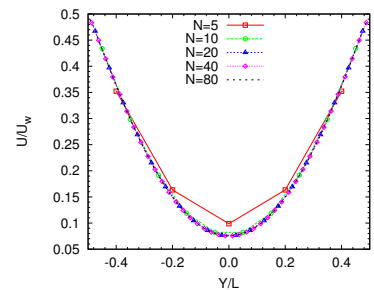

(a)

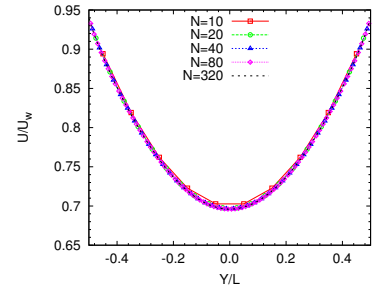

(b)

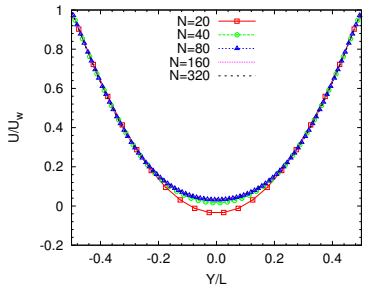

(c)

Figure 27: Velocity profiles in the impulsive start problem. (a) $\mathrm{Kn}=10$, at time $t=0.4 t_{c}$. (b) $\mathrm{Kn}=0.1$, at time $t=4 t_{c}$. (c) $\mathrm{Kn}=0.001$, at time $t=40 t_{c}$.

The flow can be treated as a pseudo one-dimensional problem, as the flow fields at any time are independent of the $x$ coordinate. Uniform structured meshes with resolutions of $N \times 1$ are used in the simulation. The relatively small computation effort is also the reason to use such a test case to investigate the convergence rate of the DUGKS. Because in the investigation of spatial convergence order, the CFL number is usually kept at very low values to suppress 
the errors induced by time integration, and very fine meshes have to be used. At such condition, simulating a full two-dimensional problem would be extremely expensive.

The $L_{2}$ errors the horizontal velocity field against the reference solutions are computed at three successively refined meshes $N_{0}, N_{1}=2 N_{0}, N_{2}=4 N_{0}$ at time $t=t_{e}$. For $\mathrm{Kn}=10, N_{0}=5, t_{e}=0.4 t_{c}$. For $\mathrm{Kn}=0.1, N_{0}=10, t_{e}=4 t_{c}$. For $\mathrm{Kn}=0.001, N_{0}=20, t_{e}=40 t_{c}$. The $L_{2}$ error is defined as

$$
\|\delta u\|_{2}^{i}:=\frac{\sum_{j=1}^{N_{i}}\left\|u\left(y_{j}, t_{e}\right)-u^{*}\left(y_{j}, t_{e}\right)\right\|_{2}}{\sum_{j=1}^{N_{i}}\left\|u^{*}\left(y_{j}, t_{e}\right)\right\|_{2}}
$$

where $u^{*}\left(y_{j}, t_{e}\right)$ is the reference solution at the grid points and is linearly interpolated from the solution obtained at a much finer mesh with mesh size $N_{m} \times 1$. The reference solutions are assumed to be the approximated accurate solutions. $N_{m}$ are 80, 320 and 640 for $\mathrm{Kn}=10,0.1$ and 0.001 , respectively. Using two different mesh sizes $N_{i}$ and $N_{i+1}$, the convergence order can be estimated by

$$
E_{N_{i}}^{N_{i+1}}=\frac{\log \left(\|\delta u\|_{2}^{i} /\|\delta u\|_{2}^{i+1}\right)}{\log \left(N_{i+1} / N_{i}\right)} .
$$

The overall convergence order $n$ is linearly fitted from $\left(\log \|\delta u\|_{2}^{i}, \log N_{i}\right)$. We expected second convergence using the current CFL condition with very low CFL numbers. For Kn $=10$, the velocity grid is a set of uniform spaced $201 \times 201$ points in the range of $\left[-4 \sqrt{2 R T_{\infty}}, 4 \sqrt{2 R T_{\infty}}\right] \times\left[-4 \sqrt{2 R T_{\infty}}, 4 \sqrt{2 R T_{\infty}}\right]$. Rectangular rule is used for numerical integration in velocity space. Using of such a fine grid in the velocity space is to minimize the ray effect at high Knudsen number. For $\mathrm{Kn}=0.1$ and $0.001,28 \times 28$ points and $8 \times 8$ points half-range Gauss-Hermit quadrature rules are used respectively. The time step size is fixed for simulation at each mesh, and Eq. 28 is replaced by $\Delta t=\alpha \Delta x /\left(10 \sqrt{2 R T_{\mathrm{w}}}\right)$ where $\alpha=0.01$ is the CFL number for all of the above configurations. The gradient limiter function is not applied for accuracy consideration.

The errors and convergence orders are listed in Table.1. The velocity profiles across the channel at each Kn are shown in Fig. 27. From Table. 1. we can 
observe that the convergence order is nearly 2 or even higher. The convergence order is independent of the Knudsen number.

\section{Concluding remarks}

380

In this paper, the DUGKS based on the Shakhov model 20] is extended to unstructured meshes. The key feature of the DUGKS is the discrete characteristic solution of the kinetic equation which is used in the modeling of the evolution of distribution function at a cell interface. Due to the coupled treatment of particle collision and transport processes, the method has the asymptotic preserving (AP) properties for capturing the Navier-Stokes solutions in the continuum flow regime. Linear reconstruction and gradient limiter are employed in the reconstruction to attain a second-order accuracy of the DUGKS.

The performance of the proposed method is explored by several examples ranging from low speed micro flows to hypersonic rarefied flows. In the transitional and slip regimes, good agreements between the results of DUGKS and DSMC solutions are observed. In the continuum regime, the DUGKS results agree well with the benchmark solutions of the Navier-Stokes equations. The second order spatial convergence order and uniform stability of the DUGKS have also been verified numerically. Thus the AP property of the DUGKS for the Navier-Stokes limit is validated. This property is important for flow that involves both continuum and rarefied regimes. As the mesh size in the continuum regimes can be much larger than the particle mean free path, the overall computational cost for the DUGKS can be largely reduced in comparison with the DSMC method and the traditional DVM. Since the DUGKS is a direct mod-

eling multiscale method 31, as the mesh size and time step size become larger than the particle mean free path and mean collision time, the physical solutions will not be sensitive to individual particle collision anymore. Therefore, in such a situation, the Shakhov model based DUGKS is accurate enough and can be faithfully used in real engineering applications. The current paper also presents a test case with two-cavities for the capturing of multiple scale flow physics. It 
is highly recommended to use this test to validate any claimed AP scheme for the gas dynamics.

\section{Acknowledgments}

We would like to thank Dr. Pubing Yu and Dr. Songze Chen for helpful China (Grant No. 51125024), the Fundamental Research Funds for the Central Universities (Grant No. 2014TS119), and the Hong Kong Research Grant Council with grants $(621011,620813,16211014)$.

\section{References}

[1] G.A. Bird, Molecular Gas Dynamics and the Direct Simulation of Gas Flows, Clarendon, Oxford, 1994.

[2] J. Fan, C. Shen, Statistical simulation of low-speed rarefied gas flows, J. Comput. Phys., 167 (2001) 393-412.

[3] T.M. Homolle, N.G. Hadjiconstantinou, A low-variance deviational simulation Monte Carlo for the Boltzmann equation, J. Comput. Phys., 226 (2007) 2341-2358.

[4] J.E. Broadwell, Study of rarefied shear flow by the discrete velocity method, J. Fluid. Mech., 19 (1964) 401-414.

[5] H. Cabannes, The discrete velocity Boltzmann equation, Lecture notes University of California, Berkeley, 1980.

[6] T. Inamuro, B. Sturtevant, Numerical study discrete-velocity gases, Physics of Fluids A, 2(1990) 2196-2203.

[7] V.V. Aristov, Direct Methods for Solving the Boltzmann Equation and Study of Nonequilibrium Flows, Springer Science \& Business Media, 2001. 
[8] L. Mieussens, Discrete-velocity models and numerical schemes for the Boltzmann-BGK equation in plane and axisymmetric geometries, J. Comput. Phys., 162 (2000) 429-466.

[9] Z.H. Li, H.X. Zhang, Gas-kinetic numerical studies of three-dimensional complex flows on spacecraft re-entry, J. Comput. Phys., 228 (2009) 11161138.

[10] M. Bennoune, M. Lemou, L. Mieussens, Uniformly stable numerical schemes for the Boltzmann equation preserving the compressible NavierStokes asymptotics, J. Comput. Phys., 227 (2008) 3781-3803.

[11] F. Filbet, S. Jin, A class of asymptotic-preserving schemes for kinetic equations and related problems with stiff sources, J. Comput. Phys., 229 (2010) 7625-7648.

[12] S. Jin, Asymptotic preserving (AP) schemes for multiscale kinetic and hyperbolic equations: a review, in: Lecture Notes for Summer School on Methods and Models of Kinetic Theory (M\&MKT), Porto Ercole, Grosseto, Italy, 2010, Riv. Mat. Univ. Parma 3 (2012) 177216.

[13] S.Z. Chen, K. Xu, A comparative study of an asymptotic preserving scheme and unified gas-kinetic scheme in continuum flow limit, J. Comput. Phys., 288 (2015) 52-65.

[14] K. Xu, J.C. Huang, A unified gas-kinetic scheme for continuum and rarefied flows, J. Comput. Phys., 229 (2010) 7747-7764.

[15] K. Xu, J.C. Huang, An improved unified gas-kinetic scheme and the study of shock structures, IMA J. Appl. Math., 76 (2011) 698-711.

[16] J.C. Huang, K. Xu, P.B. Yu, A unified gas-kinetic scheme for continuum and rarefied flows II: multi-dimensional cases, Commun. Comput. Phys., 12 (2012) 662-690. 
[17] J.C. Huang, K. Xu, P.B. Yu, A unified gas-Kinetic scheme for continuum and rarefied flows III: microflow simulations, Commun. Comput. Phys., 14 (2013) 1147-1173.

[18] K. Xu, A gas-kinetic bgk scheme for the NavierStokes equations and its connection with artificial dissipation and Godunov method, J. Comput. Phys., 171 (2001) 289-335.

[19] Z.L. Guo, K. Xu, R.J. Wang, Discrete unified gas kinetic scheme for all Knudsen number flows: low-speed isothermal case, Phys. Rev. E, 88 (2013) 033305 .

465 [20] Z.L. Guo, R.J. Wang, K. Xu, Discrete unified gas kinetic scheme for all Knudsen number flows. II. Thermal compressible case, Phys. Rev. E, 91(2015) 033313.

[21] P. Wang, L.H. Zhu, Z.L. Guo, K. Xu, A comparative study of LBE and DUGKS methods for nearly incompressible flows, Commun. Comput. Phys., 17 (2015) 657-681.

[22] L.H. Zhu, P. Wang, Z.L. Guo, A comparative study of LBE and DUGKS methods for nearly incompressible flows, Commun. Comput. Phys., 17 (2015) 657-681.

[23] E.M. Shakhov, Generalization of the Krook kinetic relaxation equation, Fluid Dyn., 3 (1968) 95-96.

[24] G.A. Bird, The DSMC method, CreateSpace Independent Publishing Platform, USA, 2013.

[25] J.Y. Yang, J.C. Huang, Rarefied flow computations using nonlinear model Boltzmann equations, J. Comput. Phys., 120 (1995) 323-339.

[26] V. Venkatakrishnan, Convergence to steady state solutions of the Euler equations on unstructured grids with limiters, J. Comput. Phys., 118 (1995) 120-130. 
[27] T. Ohwada, On the construction of kinetic schemes, J. Comput. Phys., 177 (2002) 156-175.

485

[30] T.J. Scanlon, E. Roohi, C. White, M. Darbandi, J.M. Reese, An open source, parallel DSMC code for rarefied gas flows in arbitrary geometries, Comput. Fluids, 39 (2010) 2078-2089.

[31] K. Xu, Direct Modeling for Computational Fluid Dynamics: Construction 495

[28] B. John, X.J. Gu, D.R. Emerson, Effects of incomplete surface accommodation on non-equilibrium heat transfer in cavity flow: A parallel DSMC study, Comput. Fluids, 45 (2011) 197-201.

[29] U. Ghia, K.N. Ghia, C.T. Shin, High-Re solutions for incompressible flow using the Navier-Stokes equations and a multigrid method, J. Comput. Phys., 48 (1982) 387-411. and Application of Unified Gas-Kinetic Schemes, World Scientific Publishing, 2015. 
LaTeX Source Files
Click here to download LaTeX Source Files: latex_source_files.zip

LaTeX Source Files
Click here to download LaTeX Source Files: latex_source_files.zip

Click here to download LaTeX Source Files: latex_source_files.zip

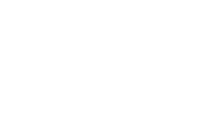

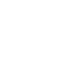

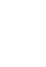
(1) (1) (1) (1) (1) (1) 更 (1) . . . . . . . . . . . . . 